ISSN: 1130-3743

\title{
TEORÍA Y PRÁCTICA DE LA ACCIÓN SOCIOEDUCATIVA
}

\author{
Theory and practice of socio-educational action
}

\author{
Théorie et pratique de l'action socio-pédagogique
}

\author{
Carmen Pereira DomíngueZ*, Jordi Solé Blanch** y Clara Isabel Fernández \\ RODICIO**** \\ * Universidad de Vigo. Facultad de Ciencias de la Educación. Departamento \\ de Análisis e Intervención Psicosocioeducativa. Avda. Castelao, s/n. Campus \\ universitario de Orense. 32004 Orense (España). Correo-e: mcdguez@uvigo.es \\ ** Universitat Oberta de Catalunya. Estudis de Psicologia i Ciències de l'Educació. \\ Rambla de Poble Nou, 156 (22@). 08018 Barcelona (España). \\ Correo-e: jsolebla@uoc.edu \\ **:* Universidad de Vigo. Facultad de Ciencias de la Educación. Departamento \\ de Didáctica, Organización Escolar y Métodos de Investigación. Avda. Castelao, \\ $s / n$. Campus universitario de Orense. 32004 Orense (España). \\ Correo-e: cirodicio@uvigo.es.
}

Fecha de recepción: marzo de 2012

Fecha de aceptación definitiva: julio de 2012

Biblid [(1130-3743) 24, 2-2012, 177-195]

\section{RESUMEN}

El objetivo de este artículo es presentar una propuesta formativa que permita practicar la teoría en la formación de profesionales del campo social y educativo. Con ella justificamos la necesidad de vincular elementos teóricos, metodológicos y éticos con las situaciones concretas que se producen en el mundo laboral. El estudio de casos a través del cine nos sirve para ejemplificar, en esta ocasión, conceptos relacionados con los psicodinamismos del trauma infantil por abuso y violencia intrafamiliar y las formas de abordarlo. Para el desarrollo y validación de esta propuesta, se convocó a dos Grupos Focales de Expertos (GFE) con los que se analizó la película 
Precious, que sirvió como estudio de caso. A partir de las ideas más significativas que surgieron de la discusión, se analizan diferentes aspectos en torno a las prácticas profesionales y los modelos de trabajo social y educativo. Se concluye que el visionado de este filme y la discusión de las coordenadas que surgen de su análisis pueden ser útiles como material complementario al académico en la formación de futuros profesionales del campo socioeducativo.

Palabras clave: violencia intrafamiliar, abuso infantil, estudio de casos, cine, educación social, trabajo social, teoría y práctica profesional, deontología.

\section{SUMMARY}

The aim of this paper is to present a training proposal to allow the realization of the training of professionals in both social and educational ways. With it we justify the need to link the theoretical, methodological and ethical actual situations occurring in the workplace. The case of study through the movies allows illustrating, this time, concepts related to childhood trauma psychodynamics of abuse and domestic violence and ways to address it. For the development and validation of this proposal, we convened two Expert Focus Groups (EFG) analyzed the movie Precious, which served as case of study. From the most significant ideas that emerged from the discussion, we analyze different aspects related to professional practices and models of social work and education. We conclude that by watching this movie and with the discussion of the coordinate guidelines that arise from their analysis may be useful as supplementary material to the academic training of future professionals into the socio-educational field.

Key words: domestic violence, child abuse, case of study, movie, social education, social work, theory and professional practice, ethics.

\section{SOMMAIRE}

Le but de cet article est de présenter une proposition de formation pour permettre à la théorie à la pratique dans la formation des professionnels sociaux et éducatifs. Avec elle on va justifier la nécessité de relier les situations théoriques, méthodologiques et éthiques réels que se produisent dans le lieu de travail. L'étude de cas à travers du cinéma on va servir pour illustrer, cette fois, les concepts liés á une psycho dynamique traumatisme de l'enfance causés par l'abus et la violence domestique et les moyens pour y remédier. Pour le développement et la validation de cette proposition, nous avons convoqué deux groupes d'experts pour analyser le film Precious, ce film a servi comme étude de cas. A partir des idées les plus importantes de la discussion, nous analysons différents aspects liés aux pratiques professionnelles et des modèles de travail social et éducatif. Nous concluons qu'avec la visualisation du film et la discussion sur les coordonnées de cette analyse sera utile comme matériel complémentaire pour la formation académique des professionnels du terrain socio-éducatif.

Mots clés: la violence domestique, la maltraitance des enfants, des études de cas, le cinéma, l'éducation sociale, travail social, la théorie et la pratique professionnelle, l'éthique. 


\section{TEORIZAR LA PRÁCTICA PROFESIONAL}

Hoy día, plantear una formación profesionalizadora en el ámbito socioeducativo no puede regirse tan sólo por un cuerpo teórico estructurado a partir de diferentes disciplinas o materias. Tal y como sostienen García y Sáez (2011, 2), "los profesionales trabajan con problemas contingentes y situados" que requieren formas de investigar y enseñar "capaces de insertarse en el régimen cotidiano de experiencias y problemas vivenciados por las personas y los profesionales". La teoría aislada, pues, no enseña una profesión. Son las situaciones concretas en las que se ponen en juego las habilidades y competencias profesionales las que permiten pensar acerca de las funciones y tareas propias de cualquier profesión. Sin embargo, ¿cómo trasladar y/o recrear en los procesos formativos esas "situaciones concretas" que se producen en los contextos donde se trabaja?

Una respuesta rápida nos lleva a pensar en el Prácticum como el momento idóneo en el que los futuros profesionales pueden visualizar las competencias, funciones y tareas propias de cualquier profesión (López y Campillo, 2009). Esto es así cuando el Prácticum se organiza adecuadamente. Pero nosotros entendemos que existen otros momentos, que en la formación académica se pueden plantear problemas vinculados a situaciones y temáticas concretas que permitan ensayar "procedimientos de abstracción" capaces de vincular elementos teóricos, metodológicos y éticos en torno a las prácticas profesionales.

Dicha forma de concebir el trabajo académico hace que la supuesta dualidad entre teoría y práctica resulte artificiosa. Ni la práctica puede concebirse como una aplicación de la teoría, ni la teoría puede encontrar una única fuente de inspiración en la práctica. Hace unos años, Clemente (2007, 27-28) nos recordaba en un artículo publicado en esta misma revista que la relación teoría-práctica ha sido siempre compleja. Según se interprete desde posiciones idealistas, la teoría es entendida como un conocimiento desinteresado, sin sentido práctico ni aplicado. Desde la perspectiva marxista, antítesis del idealismo, la búsqueda del conocimiento, e incluso de la verdad, sólo tiene lugar en la praxis. Finalmente, para la visión positivista, imperante en algunos círculos universitarios, que a menudo tiende a confundir el método con el objeto, el valor de la teoría reside en sus aplicaciones, en sus resultados prácticos, que acostumbran a quedar reducidos a una descripción superficial de la realidad social. En otro artículo, Gil (2011) ofrece una salida a la hora de establecer la relación entre la teoría y la práctica en el pensamiento pedagógico. No se trata tanto de comprobar las prácticas educativas como expresión operativa de concepciones pedagógicas, ni tan sólo de calibrar la aportación de las diferentes disciplinas que generan conocimiento en educación, sino de pensar en torno a aquellos problemas en los que, con respecto a la práctica, se pone en juego la relación educativa en sí misma.

Más allá de este debate, García y Sáez (2011, 4) nos advierten, siguiendo el trabajo de Foucault (1992), que "la teoría no expresa, no traduce, no aplica una práctica, sino que ella misma es una práctica", por lo que se puede afirmar que 
existe una "práctica de la teorización sujeta a tradiciones sociales y epistemológicas, a rutinas, órdenes e intereses de una comunidad académica, pero que puede ser liberada hacia otros destinos y encontrar otros marcos para su recreación, actualización y socialización". La teoría debe concebirse, entonces, como una caja de herramientas, cuya utilidad dependerá del uso que hagan las personas y los fines buscados. Tomando en cuenta esta posición, estos mismos autores resaltan varios principios que hemos adaptado a los propósitos de este artículo:

- Teoría y práctica se refieren a distintas estrategias o procedimientos para generar y adquirir conocimiento.

- La teoría debe orientarse hacia la comprensión y resolución de problemas vinculados con el campo profesional. Es decir, serán las propias características del problema las que orienten la selección/acomodación de los "Conocimientos teóricos" válidos para enfrentarlos y resolverlos.

- No hay teoría aislada. La teoría sólo existe en la medida que se usa. El discurso teórico debe entenderse como acción antes que como paquete de contenido. Teorizar, igual que analizar o pensar, es una práctica. En las profesiones del campo social y educativo, esa práctica tiene como objeto la construcción teórica y metodológica de las prácticas profesionales (Moyano, 2012), así como la interrogación ética y crítica de los efectos que producen en los sujetos y contextos en los que se trabaja (Núñez, 2003; Canimas, 2006; Campillo y Sáez, 2012).

Por todo ello, se hace necesario incorporar a las actividades docentes otras formas de trabajar la teoría. En este artículo presentamos una de ellas surgida de un trabajo de investigación en torno al estudio de casos a través del cine en la formación de profesionales del campo social y educativo (educadores sociales, pedagogos, psicopedagogos y trabajadores sociales, principalmente). Lo que sigue a continuación pretende ofrecer un ejemplo en el que la práctica de teorizar integra en la docencia una forma de trabajar que se da en el mundo profesional, hecho que viene a demostrar que la articulación entre investigación, docencia y proyección socioprofesional de la universidad es del todo deseable.

\section{El ESTUdio de CASOS A TRAVÉS DEL CINE}

El empleo del "estudio de casos" como estrategia analítica de investigación es un método ampliamente utilizado en las ciencias sociales. Surgido del ámbito de la investigación médica y psicológica, donde se usa para desarrollar el análisis minucioso de un proceso individual que explica la dinámica y la patología de una enfermedad, este método supone que es posible conocer un fenómeno partiendo de la explicación intensiva de un solo caso (Becker, 1979, 384). En el marco docente en el que desarrollamos nuestra actividad formativa, el objetivo que nos proponemos en torno al estudio de casos es de tipo descriptivo (intentar especificar lo que sucede con un caso particular) y explicativo (facilitar la interpretación). De 
esta forma logramos la construcción de un modelo mental general de la situación, facilitando herramientas cognitivas que favorezcan la creación del conocimiento, alcanzando así un aprendizaje significativo (Pérez, Martín, Arratia y Galisteo, 2008). Mediante la participación e implicación activa de los alumnos se posibilita la adquisición de una capacidad práctica que favorece el desarrollo de competencias.

La formación de profesionales vinculados al campo socioeducativo exige la necesidad de introducir métodos cualitativos de investigación que permitan ser aplicados en la futura realidad profesional. Trabajar con casos prácticos que puedan plantearse en el mundo laboral facilita que los estudiantes aprendan a enfrentarse a problemáticas similares a las que se encontrarán a lo largo de su carrera profesional. De este modo se dota el aprendizaje de significado y funcionalidad, estableciendo puentes entre la vida profesional y la académica, contribuyendo asimismo al incremento de competencias (Stake, 1998; Coll, Mauri y Onrubia, 2008).

Llevamos tiempo investigando de esta forma desde nuestros ámbitos formativos y académicos. Nos guía una preocupación por mejorar la motivación de los estudiantes y fomentar un aprendizaje significativo capaz de enriquecer sus experiencias (García del Dujo y Mínguez, 2011). En el proceso educativo, la representación de una situación de la realidad como base para la reflexión y el aprendizaje ofrece la oportunidad a quienes participan en su análisis de involucrarse y comprometerse tanto en la discusión del caso como en el proceso grupal para su reflexión.

El estudio de casos se puede tratar desde modelos metodológicos que permiten el uso de recursos muy diversos, ya que puede presentarse de forma narrativa o audiovisual (Castejón y Navas, 2009; Bermejo, 2005). Nosotros hemos optado por hacerlo a través del cine, teniendo en cuenta los hábitos culturales y las nuevas formas de aprendizaje y adquisición del conocimiento de las jóvenes generaciones. En esta línea, defendemos las potencialidades educativas y formativas de la imagen y los medios audiovisuales (en especial del cine) por lo que se refiere a la sensibilización, la motivación, el desarrollo de aprendizajes (sobre todo en sus dimensiones contextuales, vivenciales y sociales) y la construcción del conocimiento de los estudiantes universitarios (Pereira y Valero, 2010). Las imágenes en sentido genérico, y el cine en particular, representan indudables fuentes de conocimiento con formas discursivas propias que la cultura académica tiene que saber incorporar.

El cine y las ciencias humanas (en particular aquellas que se dedican al estudio de la mente y el ser humano) han estado inextricablemente ligadas entre sí (Gabbard, 2001). Así, en la década de los años setenta empezó a emplearse formalmente como recurso pedagógico. Se ha considerado a Wegner, en el año 1977, como el pionero en la utilización de este medio para ejemplificar conceptos complejos relacionados con las ciencias humanas (Champoux, 1998).

A partir de las décadas de los años ochenta y noventa, el uso del cine comercial en la enseñanza de asignaturas relacionadas con la filosofía (Cabrera, 2006), la psiquiatría (Shortland, 1987), la enfermería (Carper, 1999), la psicología (Gabbard, 2001), la educación en valores (Mitry, 1978) y la pedagogía juvenil (Giroux, 
TEORÍA Y PRÁCTICA DE LA ACCIÓN SOCIOEDUCATIVA

2003) comenzó a florecer de modo vertiginoso. El cine, con su poder audiovisual, su influencia a la hora de transmitir emociones y su capacidad para mimetizar la vida real, se convierte en un medio excelente para favorecer un tipo de aprendizaje capaz de desarrollarse desde la propia experiencia vital (Touriñán, 2010).

Ya en el nuevo milenio, se acuña el término de "cinema-educación" (Alexander, 2002), y el uso del cine como recurso educativo no se cuestiona, sino que más bien se promueve desde distintos campos del saber (por ejemplo, en nuestro caso, en la formación de educadores, pedagogos y trabajadores sociales, Pereira, 2005; Solé, Gómez y Gimeno, 2007). En este sentido, el cine puede convertirse en un aliado pedagógico muy válido a la hora de plantear estudios de caso desde los que promover la reflexión y el análisis sobre los principales temas teóricos y prácticos que se derivan de las situaciones estudiadas. Es lo que intentaremos demostrar a continuación, exponiendo un ejemplo en el que se aborda el fenómeno de la violencia intrafamiliar y el abuso infantil utilizando como estudio de caso la película Precious ${ }^{1}$.

\section{CAPACITACIÓN PROFESIONAL PARA AFRONTAR LA VIOLENCIA Y EL ABUSO INTRAFAMILIAR}

El objetivo del estudio de caso que aquí presentamos consiste en mostrar el fenómeno de la violencia y el abuso intrafamiliar con una doble finalidad: por un lado, realizando un acercamiento teórico y conceptual a partir del caso que nos plantea la película y, por el otro, reflexionando sobre las prácticas profesionales que se pueden desarrollar en el campo de la educación y el trabajo social.

Podríamos afirmar que, de todos los fenómenos que se observan en la clínica y el trabajo social, el del abuso sexual infantil es uno de los más difíciles de testificar. Escuchar el dolor, la angustia, y sobre todo la vergüenza que suele producir el haber sufrido abusos de un ser querido, hace que los profesionales tiendan a evitar el trabajo sincero y profundo con estas víctimas. Al respecto, Campo-Redondo (2008) ha encontrado que las historias de violencia intrafamiliar generan un conflicto emocional en los profesionales y tienden a sorprender a quien las escucha, al tiempo que pueden llegar a movilizar sentimientos negativos y de repudio hacia los victimarios ${ }^{2}$.

1. Título original: Precious, dirigida por Lee Daniels y estrenada en el Festival de Cine de Sundance, en febrero de 2009. Está basada en la novela de SAPPHiRE (1998) Push. Barcelona, Anagrama. Para una mejor comprensión del texto se recomienda el visionado de la película.

2. En España, en el año 2006 un porcentaje del 4\% de niños de 8 a 17 años han sido víctimas de algún tipo de maltrato en la esfera familiar (Informe del Centro Reina Sofía, 2011). Concretamente, el estudio realizado por GibB, CHELMINSKI y ZIMMERMAN (2007) con pacientes psiquiátricos externos concluye que los diagnósticos de depresión mayor fueron significativamente relacionados con las 
Por otro lado, debemos reconocer que la enseñanza de los efectos y los psicodinamismos del abuso sexual infantil no es una tarea fácil de poner en práctica ${ }^{3}$. Conviene que la comprensión y explicación de la violencia que genera el abuso infantil sea estudiada no sólo desde conceptos teóricos. El dolor, la vergüenza, la indignación y la impotencia que genera el abuso no se capta completamente con conceptos sencillos o tomados del sentido común; de igual modo, las "coordenadas" teóricas que iluminan el fenómeno son muchas veces complicadas de "aprehender". Por esta razón, muchos autores (tales como Hudock y Gallagher, 2001 y Shepard y Brew, 2005) han destacado la importancia de incluir vivencias con casos en el entrenamiento de profesionales que atiendan esta problemática, de modo que aprecien desde el inicio de su formación el sentido de lo que significa ser una víctima de abuso. Sin embargo, sabemos que no siempre los docentes tenemos acceso directo a casos reales para incorporarlos en la formación académica de futuros profesionales. Es aquí donde la utilización de medios alternativos, como el cine y su posterior análisis, nos sirve de herramienta, para así cubrir esta necesidad manifestada por expertos en la formación de profesionales de la salud, la educación y el trabajo social. Se podría decir entonces que el cine tiene la capacidad de ofrecer una visión condensada de la vida, y aunque no puede aprehenderla en su totalidad, es capaz de reflejar múltiples situaciones del ser humano en toda su riqueza existencial.

\section{BREVES CONSIDERACIONES METODOLÓGICAS}

Por este motivo, nos propusimos diseñar una experiencia de investigación que permitiera desarrollar un material docente capaz de mostrar la complejidad del abuso infantil y las formas de abordarlo, y lo hicimos a través de la proyección y reflexión de una película comercial. Lo que expondremos a continuación trata de documentar esa experiencia con dos Grupos Focales de Expertos (GFE) ${ }^{4}$ con quienes se analizó la película Precious que, tal y como ya hemos avanzado, tomamos

denuncias de abuso emocional en la infancia y el abuso físico o sexual. El maltrato más frecuente es la negligencia (cuando las necesidades básicas del niño no están cubiertas), que supone aproximadamente el $50 \%$ de los malos tratos, seguido del maltrato psíquico (27\%), el físico (11\%) y el sexual (6\%) (Pou, 2010).

3. Por psicodinamismos entendemos los aspectos psicológicos que subyacen a una conducta o fenómeno en particular, especialmente aquellos que se relacionan con los aspectos inconscientes de la misma.

4. El concepto de Grupo Focal de Expertos (GFE) consiste en una conversación con un conjunto de especialistas en un determinado tema o que tienen mucha experiencia sobre cierta área, en este caso, el abuso infantil y la violencia intrafamiliar (VARGAS e IBÁÑEZ, 2008). 
como estudio de caso 5 . Nuestro propósito fue el de obtener las "coordenadas" ${ }^{6} \mathrm{O}$ unidades de significado que permitieran explicar de forma comprensible y sobre todo vivencial (Pereira, 2009), conceptos relacionados con la fenomenología del trauma psicológico y los posibles abordajes y prácticas profesionales que se pueden desarrollar desde el trabajo social y educativo ${ }^{7}$.

El propósito fundamental es comprender la particularidad del caso (la vida de Precious), para entender todas las partes que lo componen (su familia, el grupo de iguales, los técnicos de los servicios sociales) y las relaciones establecidas entre los distintos subsistemas (familia, escuela, servicios sociales) y que forman un todo (Yin, 1989; Stake, 1995).

El criterio seguido para seleccionar a los integrantes de los GFE consistió en que estos profesionales tuviesen experiencia en el ámbito de la infancia y la juventud, la salud mental, la educación y el trabajo social ${ }^{8}$.

A las personas integrantes de esta experiencia se las congregó con el fin de proyectar la película en grupo, y una vez visionada, comentar sus impresiones y ubicar

5. La historia de Clarencee Jones (Precious), es la de una adolescente de dieciséis años, negra, casi analfabeta, que espera su segundo hijo. El primero lo tuvo a los doce años, una niña con síndrome de Down a la que apodan con el nombre de Mongo, después de que la violara su propio padre. Precious vive en Harlem con su madre (Mo’Nique), en Lenox Avenue, una de las zonas más marginales del barrio. El filme nos presenta una madre vulgar y cruel, embrutecida por el abandono y la desidia, cuya única ocupación diaria es ver la televisión, comer lo que Precious le prepara y mantener las prestaciones sociales a costa de su hija y su nieta, mientras la maltrata y la hace culpable de las propias agresiones sexuales que sufre por parte de su padre, acusándola de robarle a su marido y tratándola de un modo humillante. Precious tiene que abandonar la escuela a causa de su embarazo. El centro escolar es su singular atadura al mundo real, el único lugar por el que siente algún interés y en el que consigue, aun siendo invisible, dejar el entorno en el que vive. Sin embargo, y a causa de su embarazo, Precious debe salir del instituto mientras es derivada a un centro educativo para casos especiales. En él iniciará el camino de la recuperación. Allí encontrará a la Srta. Rain (Paula Patton), una maestra joven y lesbiana que le hará sentirse alguien reconocido mientras le ofrece la oportunidad de aprender. Junto con el resto de compañeras de la clase, otras chicas con vidas vulnerables, y con la ayuda de la trabajadora social (Mariah Carey) y de un enfermero (Lenny Kravitz), que serán capaces de verla más allá de la simple apariencia, Precious recuperará su autoestima y comenzará su ascenso desde los infiernos.

6. Tomamos el concepto de coordenada de uno más amplio denominado concepto-imagen, acuñado por el filósofo del cine Julio CABRERA (2006, 21). El concepto-imagen es definido por este autor como encadenamientos de los que se vale el director de una obra cinematográfica para transmitir una idea o un conocimiento, pero sin querer trazarla completamente. Así, los conceptos-imagen del cine son como caminos que afirman algo sobre el mundo con pretensiones de verdad y universalidad, en el sentido de que el cine expresa algo que podría ocurrirle a cualquiera, en vez de ser algo que acontece necesariamente a todos.

7. Nos parece oportuno referenciar la experiencia de intervención pedagógica a través del cine, a propósito del Programa Respeto, llevado a cabo hace unos años en un centro penitenciario con un grupo de presidiarias. Una vez vistas algunas de las películas programadas, parte de estas mujeres reflejaron en el fórum establecido sus vivencias como víctimas de abuso intrafamiliar (PEREIRA, 2009).

8. Sumando los componentes de ambos grupos, se contó con la presencia de los siguientes profesionales: 3 psicopedagogos, 4 psicólogos, 5 educadores sociales, 2 maestras, 1 filósofa, 5 médicos, 1 sociólogo, 2 trabajadores sociales, 1 pedagogo y 2 estudiantes. El $85 \%$ de los profesionales son mujeres. 
las coordenadas que dieran sentido al filme. Se hicieron dos sesiones - una para cada grupo- que se grabaron en su totalidad (previa autorización de los presentes).

\section{ANÁlisis y pROpuestas de trabajo de los GFe a PROPÓsito de PRECIOUS}

A continuación presentamos un breve resumen de las coordenadas o unidades de significado que emergieron de la discusión de los dos GFE a partir del análisis de la película (Carmona, 1991; Glenn, 2001; Fernández y Pereira, 2010 y Marín, 2011). Posteriormente, se realiza una triangulación con algunos conceptos teóricos a fin de situar el tema de la violencia y el abuso intrafamiliar y su conexión con las prácticas profesionales y modelos de trabajo socioeducativos más efectivos.

\section{a) Tener un lugar en el mundo}

Precious (Preciosa) es el nombre adjudicado al personaje principal del filme. Desde el título de la película se nos da una idea de la importancia del nombre de la protagonista para contar su historia. Esta identificación permite que la protagonista se identifique con algún valor resiliente en su vida. Podría hipotetizarse que su nombre simboliza la idealización que, en algún momento de la vida, tuvieron los padres de esta niña. En otras palabras, el nombre dado a la protagonista le imprimió un valor fundamental para que ésta pudiese resignificar su trauma y su vida. En toda su historia, la protagonista no ha sido apreciada como ser humano diferente a sus padres; sin embargo, su nombre no es común, por lo que cabe pensar en un primer intento de otorgarle cierta distinción, como si se tratara de un sello, una marca o una etiqueta distintiva especial. Podríamos suponer que los padres de Precious no le asignaron un lugar en el mundo; es sólo a través de su nombre que Precious puede seguir las huellas para conseguir uno y sentirlo como propio. Quizás, a través del nombre escogido por la madre, ésta demuestra haber tenido también alguna preocupación maternal primaria que salvó a la niña de la locura. Es muy probable, por lo demás, que el nombre impuesto a la pequeña haya estimulado la intensa imaginación que luego desarrolló como mecanismo compensatorio para mitigar su victimización. El nombre expresa un modelo de identificación que adquiere significados desde lo personal. En el caso de Precious tiene una carga importante de ironía: a pesar de que implica un protagonismo, que le permitiría considerarse única, exclusiva, diferente a los demás, ella no se identifica a sí misma como tal, salvo en la desdicha y la infelicidad.

\section{b) Sobrevivir al abuso}

Precious es la principal protagonista de la historia. Es una adolescente víctima del goce perverso de sus padres. El personaje de Precious viene a representar el holocausto físico y psicológico infligido a una persona inocente por quienes 
se supone que debieron protegerla y nutrirla. Este trauma marca la identidad de Precious, y no es hasta el final de la película cuando ella es capaz de retomar las riendas de su vida. Precious, además, es analfabeta, lo que metafóricamente se asocia con una incapacidad para reconocerse a sí misma. El lenguaje es lo que da forma y sentido a toda una experiencia. En un momento dado Precious verbaliza "Puedo gritar, pero aun así no existo" (34' 55"). Confrontada a los abusos de sus padres no puede traducir la defensa de su integridad en palabras. Éstas no hacen más que acumularse en su interior, lo que permite explicar algunas de las causas que provocan, por ejemplo, su obesidad. Es a través de su propio monólogo que sirve como hilo conductor de la historia y las escenas referentes a sus fantasías donde podemos conocer su dolor como víctima de los abusos sufridos. Precious tiene una intensa vida imaginativa que la salva de caer en un vacío existencial mayor del que ya tiene. Consigue mantenerse alejada de la amarga realidad en la que vive a través de este mecanismo de defensa. Aunque ella apenas puede dimensionar el impacto de su analfabetismo, sí es consciente del estigma al que se le asocia por no saber leer ni escribir. Así, cuando la Srta. Rain, su nueva maestra en el colegio especial, le intenta enseñar, Precious responde de modo agresivo frente a su incapacidad debido a su frustración. Mientras tanto, Precious sueña con ser una bella y delgada bailarina; aunque también tiene momentos oscuros: unos adolescentes callejeros la persiguen y atormentan. Sin embargo, su única salvación consiste en refugiarse en esa intensa vida que imagina y desarrolla, aunque para ello distorsione la realidad. Esta distorsión es su refugio mientras fantasea con ser reconocida por su riqueza interior.

\section{c) La perturbación de los procesos de apego}

La madre de Precious, Mary, es representada como una matrona monstruosa, apresada en su propio destino e incapaz de hacer algo para cambiarlo. Mary estuvo recluida, y podríamos decir que a pesar de su crueldad, también puede ser considerada como una víctima, tanto de la sociedad a la que chantajea (utiliza a su hija y a su nieta para cobrar la pensión de los servicios sociales), como del padre de su hija. En el momento que la trabajadora social puede establecer una relación más o menos sincera con ella, descubrimos que detrás de esa madre despiadada se esconde una mujer invadida por el miedo, la angustia y la depresión, sin que por ello se justifique su comportamiento. Mary quedó atrapada en la perversión de su "pareja", incapaz de poner límites a sus deseos incestuosos. En lugar de reprobarlos, se muestra celosa y por ello desprecia y maltrata a Precious, su hija. Ésta, aun siendo muy pequeña, es vivida como una amenaza y la no concordancia entre la niña imaginada y la niña real, capaz de robarle a "Su hombre", acaba por perturbar los procesos de apego y favorecer la emergencia del maltrato. Precious se convierte, así, en objeto de venganza. Además, Mary no tolera la soledad, y eso da pie al abuso que ejerce sobre su hija, "prisionera" de esta figura primaria de apego “Ven a cuidar a mamá, Precious!» (22' 21”), favoreciendo su obesidad. De 
este modo, en un momento de la película, Mary verbaliza, "Era mi hombre, era mi puto hombre, era mi hombre y deseaba a mi hija y por eso la odiaba tanto" ( $1 \mathrm{~h}$ 34' 31"). Y sigue diciendo, "Ella le contó que yo le hacía... ¿quién iba a quererme? Usted que tiene un título (refiriéndose a la trabajadora social, Miss Wiess), y sabe tantas cosas, dígame ¿quién iba a quererme y hacerme sentir bien por las noches?" ( 1 h $35^{\prime}$ 04"). A través de la historia se puede inferir que Mary alguna vez tuvo sueños y quizás la capacidad de amar (al menos le puso un nombre "precioso" a su hija); alguna reminiscencia de bondad debió tener para salvar a Precious de la locura y la perversión. Una escena de la película lo refleja muy bien, cuando Precious intercala dulces recuerdos fotográficos de su infancia con su madre durante una fuerte pelea con ella al salir del hospital con su segundo bebé (1 h 3' 01”). Tal vez estos recuerdos evitarán que Precious repita la historia de su madre.

\section{d) La transmisión intergeneracional del trauma}

La abuela de Precious y madre de Mary cumple un rol menor pero relevante en la trama: cuida a Mongo, con síndrome de Down, hija de Precious, y de alguna manera le da un referente de normalidad a su nieta. Hay varias alusiones en la película que nos indican que la abuela sentía un profundo temor hacia su hija Mary. Como muestra, le dice a su nieta, "Tú no eres como tu madre" (58' 57") (haciendo alusión a que no es igual en maldad), y esto debió tener algún efecto en Precious, que la hizo diferenciarse de la crueldad de su madre. Con todo, la abuela es cómplice del maltrato que sufre Precious, pues no toma decisiones ni denuncia la situación. Asume su rol en la farsa ante los servicios sociales, aunque lo hace para proteger a la niña. De igual modo, su personaje sirve para introducir la idea de la importancia del trauma intergeneracional, y las consecuencias de no poder asumir la maternidad y los límites en la crianza de los hijos. Haciéndose cargo de la hija de Precious, como hacen tantas "abuelas acogedoras", establece un intento de reparar lo que pudo "no haber funcionado" en el cuidado y educación de su propia hija, con quien no puede reconocerse. Su resignada aceptación de las diferentes formas de maltrato que se producen en el seno familiar lo convierten en una experiencia organizadora de la fenomenología familiar, hecho que permite desarrollar hipótesis interpretativas en torno a las familias transgeneracionalmente perturbadas.

\section{e) El sujeto abusador}

Representada por el padre de Precious, nos hallamos ante una figura "ausente", pues en ningún momento se ve su rostro (sólo a modo de sombra y de lejos). Esta forma de representación lo convierte en un ser "amenazante". Su perversidad se hace sentir a través de toda la película. Su "ausencia" nos demuestra que el daño del victimario no sólo es físico, sino que también se corresponde con su recuerdo. El modelo del padre sirve para explicar la estructura de las familias sexualmente 
abusivas, caracterizadas por fronteras y roles familiares poco claros y mal definidos. "La persona que tiene que proteger es la que daña...". Precious "necesita recibir el apego de un adulto, pero el adulto abusador no deja de ser figura de apego" (Abad, 2009, 39).

El relato de Mary permite constatar la configuración del papel del padre, incapaz de diferenciar a una niña de una mujer, a una esposa de una hija. El contacto físico, la excitación, la sexualidad son una misma cosa más allá del objeto al que se dirige el deseo sexual. Su ausencia en el desarrollo del filme permite incidir en la brutalidad que sufre la protagonista de la película, pero no nos deja reconocer, como sí ocurre en el caso de Mary, los elementos más importantes que constituyen las vivencias de un hombre que ha seguido su propio proceso de individuación. Queda abierto, pues, el interrogante a la hora de intentar describir y explicar las experiencias vitales y la personalidad de los sujetos abusadores y, con él, las posibilidades terapéuticas y/o de rehabilitación.

\section{f) El vínculo educativo}

La Srta. Blue Rain (Lluvia Azul), maestra de la "escuela especial", es una de las figuras "terapéuticas" de la película, junto a la trabajadora social. Para Precious representa la madre buena, alguien con quien puede identificarse. Es a través de la relación que establece la Srta. Rain con Precious que esta última puede resignificar su vida, y establecer una narrativa diferente hacia su pasado. La Srta. Rain es quien ofrece a Precious la oportunidad de establecer un vínculo de confianza, que es el que posibilita el retorno al lugar donde se encuentran los afectos, así como expresar sus emociones, lo que le proporcionará la capacidad para sobreponerse a esta etapa traumática, fortaleciendo su resiliencia, que guiará a nuestra protagonista para continuar un desarrollo personal que se ha visto alterado por heridas muy profundas. En este sentido, la Srta. Rain imprime una nueva identidad a Precious, al considerarla un ser humano, con características propias y bondadosas. El vínculo educativo que establece con ella implica a nuestra protagonista en una dinámica positiva, desarrollando su capacidad para salir adelante y reforzada de las experiencias adversas, lo que permite que Precious pueda escapar del victimismo. Ella es quien introduce a Precious en el mundo de la lectura y la escritura, permitiéndole crear una nueva narrativa para su vida. Además, la Srta. Rain y Precious mantienen un intercambio de diarios, que bien asemeja a la actividad terapéutica que se realiza con víctimas de abuso sexual ya que le permite expresar su dolor. Al respecto, Precious comenta: "Me siento feliz escribiendo, me siento feliz en esta escuela. La Srta. Rain quiere que escribamos todos los días. Ella nos ayuda con las dudas" (51'40") y "La Srta. Rain fue la que puso una tiza en mi mano. Me hizo la reina del abecedario" (1 h 13' 24"). Estamos ante una clara demostración de que la palabra alivia y reconforta. Además, la Srta. Rain enseña a leer a Precious, y como una metáfora de la vida, le brinda una nueva lectura a la historia de la protagonista. 


\section{g) Las prácticas profesionales en el trabajo social}

La trabajadora social viene a ser el medio por el cual Precious forja su propio destino y resignifica su identidad, de víctima dependiente de la crueldad de su madre, a persona autónoma capaz de forjar su propio destino. Ella es la que facilita un espacio de diálogo que permite a las protagonistas de este drama familiar recuperar sus memorias. Es este personaje el que vehiculiza que la madre de Precious confiese su crimen y logre concienciar el horror que Precious ha vivido, al tiempo que aprueba que Precious construya su posición como madre capaz de cuidar a sus dos hijos y hacerse cargo de su destino y no se instale como víctima crónica de abuso. Su intervención es muy importante, aunque Precious llega a cuestionarla en una de las entrevistas después de haber relatado el horror sufrido. Si la Srta. Weiss no hubiera confiado en las capacidades de Precious para salir adelante, creyendo que corría el riesgo de repetir el trauma, se hubiera activado otro tipo de intervención, por ejemplo, tutelando a sus hijos y separándola de ellos.

\section{h) La importancia de las redes sociales}

Las amigas de Precious simbolizan la noción de grupo, apoyo y reconocimiento a su soledad como víctima. El amparo, la comprensión y la ayuda que recibe por parte de sus compañeras es otro de los factores que contribuyen a fortalecer la capacidad de resiliencia de nuestra protagonista. La película permite apuntar hacia esta otra forma de apoyo social que, desde el punto de vista de la intervención y el tratamiento terapéutico, deviene un buen complemento del trabajo individual. La Srta. Rain favorece esas dinámicas de grupo al entender que abren la posibilidad de nuevos aprendizajes para comunicar, actuar y reflexionar en relación con lo pasado y así "despegarse" y "descontaminarse" de lo aprendido en los procesos abusivos y maltratadores que han sufrido sus alumnas.

\subsection{Triangulación con algunos conceptos teóricos (I). Precious como víctima: falso self versus verdadero self}

Precious es mostrada en este filme como un ser victimizado por sus padres y por la sociedad, no sólo en las esferas psicológica y pedagógica, sino también en la corporal. Desde su nacimiento, a nuestra protagonista no le fue otorgado un lugar en el mundo, sólo el físico (de ahí su obesidad mórbida), y para el goce perverso de ambos padres. Precious, como dice ella misma, se debate entre ser objeto de goce de otros o independizarse para evitar seguir siendo una víctima de su madre.

Como toda víctima de abusos severos y repetitivos por parte de sus cuidadores más cercanos, Precious recurre a la disociación como mecanismo de defensa frente a tanto sufrimiento. Ella se aleja mentalmente de las situaciones y recuerdos más dolorosos, y se imagina blanca, bella, esbelta, dulce, y bailando con un príncipe 
que la rescata. Asimismo, crea un hada madrina (un símbolo de su deseo de tener una buena madre que la cuide y quiera) que la ama, y que le sirve para calmarse frente a su adversidad. Esta fantasía disociativa le permite mitigar el dolor psíquico por el abuso y la negligencia a la que ha sido expuesta durante toda su vida. En otras palabras, podría decirse que la fantasía salva a Precious, tanto de la locura (psicosis), como de la perversión (psicopatía). A su vez, esta fantasía hace que Precious tenga deseos, y en última instancia logre "Salvarse" y reiniciar un nuevo rumbo (al final de la película). Su fantasía es un síntoma, una respuesta que ella inventa para poder soportar el dolor.

A pesar de que la fantasía ayuda a Precious a disociar vivencias dolorosas, esto tiene un coste en su vida emocional: la protagonista pierde conciencia de ella misma para poder tolerar su tragedia. Esto se evidencia de modo significativo en la reacción que tiene frente a sus dos embarazos. En el primero, le confiesa a su maestra que ella "no sintió nada" cuando nació su primera hija, Mongo. En el segundo, en cambio, se produce un punto de inflexión al verbalizar que "esta vez sí dolió", refiriéndose al alumbramiento de Abdul, su segundo hijo. El cambio que Precious efectúa a lo largo de la película, a consecuencia de su nueva red social, así como a la influencia de la maestra y la trabajadora social, hacen que amplíe la conciencia de sí misma, incluida aquellas sensaciones dolorosas de la vida, como el hecho de alumbrar un hijo.

Siguiendo la reconocida teoría de Winnicott (1965) sobre el proceso de maduración en la infancia, podríamos afirmar que todo el sufrimiento infligido a Precious impidió que desarrollara un verdadero self y creara lo que este autor denomina "un falso self". Al respecto, este psicoanalista comenta que cuando se ha formado un self falso, sería en parte porque ha sido el niño el que ha tenido que adaptarse al medio (a la madre), en vez de ser ésta la que se adapte a las necesidades del niño. El self falso aparecería entonces como defensa.

Así, toda esta vida "adaptada" que Precious desarrolló, tanto su pasividad frente a los castigos, como su inhabilidad para leer, se corresponde con un "falso self", puesto que debió renunciar a una parte de su propia conciencia para satisfacer el goce de sus padres. Al respecto, Winnicott $(1982,177)$ nos comenta que

cuando la madre no es capaz de adaptarse bien, el niño se ve seducido a la sumisión y es un ser falso y sumiso quien reacciona ante las exigencias del medio ambiente, que parecen ser aceptadas por el niño. Por mediación de este ser falso el pequeño se construye un juego de reacciones artificiales, y por medio de introyecciones llega incluso a adquirir una ficción de realidad. El ser falso tiene una función positiva muy importante: ocultar al ser verdadero, lo que logra sometiéndose a las exigencias ambientales.

Esta idea nos lleva a la caracterización que se hace de Precious, en el sentido de que no había espacio en su vida para la espontaneidad (de ahí su obesidad) ni para la creatividad (quizás esto explique su inhabilidad para leer, como una metáfora de su incapacidad para "leerse" a sí misma, excepto en su mundo de fantasía). 


\subsection{Triangulación con algunos conceptos teóricos (II). Prácticas profesionales y modelos de trabajo social y educativo}

Ya hemos dicho en otro apartado que los casos de abuso sexual y maltrato intrafamiliar despiertan todo tipo de recelos. Más allá de la credibilidad del testimonio infantil, que ya hemos tratado, aparece la cuestión de la angustia de los propios especialistas (Pantoja, 2012). Así lo pudimos verificar durante la conversación mantenida con los GFE. Algunos de los componentes, con largas trayectorias profesionales en el campo de la protección a la infancia y la adolescencia, no pudieron evitar sentirse reconocidos en las dos trabajadoras sociales que realizan la visita de supervisión en el domicilio de la madre de Precious para comprobar que todo está en orden y poder prorrogar, así, la ayuda económica de los servicios sociales sin "querer ver nada". Monitorizar las prácticas profesionales, limitarse a seguir protocolos de actuación, son modelos de trabajo que -tal y como nos dice Ubieto (2012) - permiten reducir la angustia de los profesionales y "Soportar" su propio malestar.

A menudo, esa angustia del experto viene generada por la propia angustia del sujeto que está sufriendo una situación de abuso o malos tratos. Los profesionales no sólo detectan una necesidad, sino que deben hacer frente a una situación subjetiva, es decir, a la existencia de un sujeto que mantiene una posición particular ante lo que está viviendo. Esa posición subjetiva puede presentarse en forma de angustia, proyectando todo tipo de síntomas (en el caso que nos ocupa, depresión, obesidad, embarazos prematuros, fracaso escolar, etc.). Precious está sola, indefensa en la desesperación. Ella no realiza ninguna demanda, pero todos podemos percibir su angustia. No podemos ignorarla; sin embargo, el peligro de atender esa amargura nos confronta a la impotencia y nos puede empujar a proveer recursos substitutivos (prestaciones económicas, ayudas sociales, etc.) -como pone en evidencia la película-. En otros casos, la respuesta a esa "contaminación" subjetiva, a esa aflicción que el sujeto nos traslada, se produce por la vía del pasaje al acto, poniendo al otro en la situación de hacerse expulsar, exigiéndole, por ejemplo, contraprestaciones imposibles, con las que, indirectamente, rechazamos su demanda y la segregamos. Es lo que podría haber hecho la Srta. Weiss si hubiera exigido a Precious un trabajo estable, una vivienda fija, etc., para hacerse cargo de sus hijos. Ese pasaje al acto al que se ven expuestos muchos especialistas puede ser inadecuado, pero lógico y comprensible -tal y como señalaron diferentes miembros de los GFE- desde el punto de vista subjetivo y profesional.

Junto al debate en torno a los modelos de trabajo social, el filme también aborda la cuestión de la acción educativa. La Srta. Rain es la que logrará sacar a Precious de su analfabetismo, y lo hace no sólo porque tiene un interés por ella -así como por el resto de sus alumnas-, sino porque la base de su motivación se orienta hacia la "cultura", el deseo de enseñarle, de poner en evidencia su interés y valor. Esto es lo que favorece la educación de las muchachas que asisten a la escuela. 
La Srta. Rain abre las puertas a mundos desconocidos, «ilumina" -como dice Precious- un encuentro con algo nuevo. Asume, así, una función de causa para despertar la motivación de las jóvenes. La inquietud de la profesora Rain "causa" el interés de Precious y sus compañeras. A través de este interés pone en juego su deseo y hace posible la transferencia. Bajo la transferencia se puede sintomatizar el aprendizaje. Es decir, por la vía de la oferta cultural se puede operar una cierta regulación del síntoma?.

Mientras tanto, el diario se convierte en el intermediario más eficaz para vehiculizar esa transferencia. El diario ayuda a elaborar nuevos significados. A través de él, Precious se constituye como sujeto y logra introducir nuevas perspectivas en su mundo. La Srta. Rain ofrece recursos a Precious para "permitirle hacer" por la vía del deseo. La educación se convierte, así, en una oportunidad de cambio, una transformación en acto que proyecta a nuestra protagonista hacia otra manera de verse a sí misma y de entender el mundo. Éste es el gran mensaje que nos lanza el filme en torno a la acción social y educativa: la gran potencia del acto educativo puede hallarse cuando el agente de la educación ofrece materiales culturales que posibiliten al sujeto repensarse desde nuevas perspectivas, más allá de su vida personal y de sus heridas.

\section{A MODO DE CONCLUSIÓN}

Un filme puede generar infinitas interpretaciones y su posterior discusión puede tomar diversas vertientes. En la materia que nos ocupa, nos interesó poder articular un estudio de caso hipotético (pero según los expertos no distante de los "reales") de abuso sexual intrafamiliar a través del cine. Se trata de presentar una situación concreta que forma parte de la realidad social y laboral para poner en juego los elementos teóricos, metodológicos y éticos que contribuyen a construir una posición profesional en torno a las prácticas del trabajo social y educativo a las que esta situación convoca. La documentación precisa de lo que produjeron los Grupos Focales de Expertos (GFE) permite presentar un análisis y una forma de practicar la teoría que puede incorporarse en la formación de futuros profesionales del campo socioeducativo y la salud mental.

9. El psicoanálisis diferencia lo que podríamos llamar el síntoma patológico del síntoma que es la manera de ser de cada uno, la forma en que cada uno hace vivible la vida por medio de un modo de obtener satisfacciones. Es lo particular de cada sujeto y es la base de la diversidad. Tal y como sostiene NúÑEZ (2010), este síntoma es una forma de tratamiento de lo pulsional, es un regulador del placer que sufre tropiezos, pero que, de una manera u otra, se puede reconducir. Cuando se desregula produce el síntoma que se considera patológico. La educación social trabaja con síntomas sociales, pero hay que recordar que éstos se encarnan en sujetos concretos. El sujeto da su consentimiento cuando hay transferencia, pero la transferencia hay que causarla. Es el interés del educador lo que causará el interés del sujeto, en tanto que lo que se transmite es el deseo puesto en juego. 
Este modo de trabajar, muy propio, por lo demás, del mundo profesional, habituado a abrir espacios de debate y supervisión (Moyano, 2011), nos parece muy válido para construir un saber en torno a otros fenómenos y otros casos. La conversación entre grupos de profesionales, que después trasladamos a nuestras aulas, permite elaborar nuevas formas de representarnos las situaciones que tratamos, favoreciendo la implicación emocional a través de un medio que, como el cine, no sólo se convierte en fuente de conocimiento, sino también de nuevas experiencias vitales (Gutiérrez, Pereira y Valero, 2006).

Consideramos que el ejemplo que hemos presentado en torno a este estudio de caso tiene la suficiente validez como para ser empleado en futuros cursos, de modo tal que los estudiantes del campo social y educativo puedan tener una aproximación a la fenomenología de un caso de abuso sexual infantil y las prácticas profesionales que se pueden poner en juego para poder abordarlo. Es una forma de practicar la teoría en la formación de futuros profesionales mediante el uso del cine, el estudio de casos y la conversación interdisciplinar.

\section{REFERENCIAS BIBLIOGRÁFICAS}

ABAD, M. (2009) El abuso sexual. Comentario sobre un caso clínico. Aperturas Psicoanaliticas. Revista Internacional de Psicoanálisis, (1), 31.

Alexander, C. (2002) The Doctor: A seminal Video for Cinemaeducation. Family Medicine, $34(2), 92-94$.

BeCKer, H. (1979) Observación y estudios de casos sociales, en Sills, D. (dir.) Enciclopedia Internacional de las Ciencias Sociales, 7. Madrid, Aguilar, 384-389.

Bermejo, J. (2005) Narrativa audiovisual. Madrid, Pirámide.

Cabrera, J. (2006) Cine: 100 años de Filosofía. Una Introducción a la filosofía a través del análisis de películas. Barcelona, Gedisa.

Campillo, M. y SÁEz, J. (2012) Por una ética situacional en Educación Social. Pedagogía Social. Revista Interuniversitaria, 19, 13-36.

CAMPo-Redondo, M. (2008) Reacciones contratransferenciales en orientadores de la violencia familiar. Revista Artes y Humanidades, 9 (23), 75-98.

Canimas, J. (2006) La ética aplicada a la intervención social. Revista de Servicios Sociales y Politica Social, 73, 135-146.

Carmona, R. (1991) Cómo se comenta un texto filmico. Madrid, Cátedra.

CARPER, J. (1999) Integrating patterns of knowing in an undergraduate nursing course. Nurse Educator, 24 (4), 26-30.

CASTejón, J. L. y NAVAS, L. (2009) Aprendizaje, desarrollo y disfunciones. Implicaciones para la enseñanza en la Educación Secundaria. Alicante, Editorial Club Universitario.

Champoux, J. (1998) Film as a teaching resource. Journal of Management Inquiry, 8 (2), 240-251.

Clemente, M. (2007) La complejidad de las relaciones teoría-práctica en educación. Teoría de la Educación. Revista Interuniversitaria, 19, 25-46.

Coll, C.; Mauri, T. y OnRubia, J. (2008) Los entornos virtuales de aprendizaje basados en el análisis de casos y la resolución de problemas, en Coll, C. y Monereo, C. (comps.) Psicología de la Educación Virtual. Madrid, Editorial Morata, 213-232. 
Fernández, C. I. y Pereira, C. (2010) Cine y Transversales: Precious. Padres y Maestros, 34 (páginas centrales). Consultado el 20 de junio de 2012. http://www.upcomillas.es/ revistas/PadresYMaestros/Revi_Padr_Maes_Revi.aspx.

Foucault, M. (1992) El orden del discurso. Buenos Aires, Tusquets.

GabBard, G. (2001) Psychoanalysis and film. London, Kamac.

García, J. y SÁEZ, J. (2011) Educación Social ¿Qué formación para qué profesional? RES. Revista de Educación Social, 13, 1-14. Consultado el 20 de julio del 2012. http://www. eduso.net/res/pdf/13/esforpro_res_13.pdf.

García del Dujo, A. y Mínguez, R. (2011) Los límites de la educación en valores cívicos: cuestiones y propuestas pedagógicas. Educación XXI. Revista de la Facultad de Educación, 14 (2), 263-284.

Gib, B.; CheKminski, E. y Zimmerman, M. (2007) Childhood emotional, physical, and sexual abuse, and diagnoses of depressive and anxiety disorders in adult psychiatric outpatients. Depress. Anxiety, 24, 256-263.

GIL, F. (2011) Educación con Teoría. Revisión pedagógica de las relaciones entre la teoría y la práctica educativa. Teoría de la Educación. Revista Interuniversitaria, vol. 23, $19-43$.

Giroux, H. A. (2003) Cine y entretenimiento. Elementos para una crítica política del cine. Barcelona, Paidós.

GLenn, G. (2001) Psychoanalysis and Film. International Journal of Psychoanalysis. London, Key Papers Series.

Gutiérrez, C.; Pereira, C. y Valero, L. F. (2006) El cine como instrumento de alfabetización emocional. Teoría de la Educación. Revista Interuniversitaria, 18, 220-260.

Hudock, A. M. y Gallagher, S. A. (2001) Using movies to teach family systems concepts. The Family Journal, 9, 116-121.

Informe Del CENTRO Reina Sofía (2011) Estudio sobre Maltrato Infantil en Familia. Madrid, Ministerio de Sanidad, Política Social e Igualdad.

LÓPEZ, M. y CAMPILLO, M. (2009) Universidad y profesionales: la formación y las competencias profesionales. Universitas Tarraconensis, III época, 131-143.

Marín, F. (2011) Adolescentes y maternidad en el cine: "Juno", "Precious" y "The Greats". Comunicar, 36 (XVIII), 115-122. Consultado el 20 de junio de 2012. http//:www.revistacomunicar.com.

Mitry, J. (1978) Estética y psicología del cine. Madrid, Siglo XXI, 2 vols.

Moyano, S. (2011) La supervisión: una herramienta para la reflexión educativa, en Moyano, S. y Planella, J. (coords.) Voces de la educación social. Barcelona, Editorial Uoc, 209-218.

- (2012) Acción educativa y funciones de los educadores sociales. Barcelona, Editorial UOC.

NúÑEZ, V. (2003) Entre la tecnociencia y el tecnopoder: el desafío de mantener abierta la pregunta de las condiciones de producción de la Pedagogía Social y sus efectos. Pedagogía Social. Revista Interuniversitaria, 10, 111-122.

- (2010) El lugar de los modelos, en Núñez, V. (coord.) Encrucijadas de la educación social: orientaciones, modelos y prácticas. Barcelona, Editorial UOC, 17-68.

Pantoja, L. (2012) Deontología y código deontológico del Educador Social. Pedagogía Social. Revista Interuniversitaria, 19, 65-79.

Pereira, C. (2005) Cine y Educación Social. Revista de Educación, 338, 205-228. 
- (2009) Cine, cárcel y mujeres. Un ejemplo de creación de conocimiento.Enl@ce.Revista Venezolana de Información, Tecnología y Conocimiento, 6 (2), 39-55. Consultado el 20 de junio de 2012. http://revistas.luz.edu.ve/index.php/enlace/issue/archive.

Pereira, M. ${ }^{a}$ C. y Valero, L. F. (2010) El cine en los estudios de Educación Social como catalizador para la construcción del Espacio Europeo de Educación Superior. Enseñanza \& Teaching. Revista Interuniversitaria de Didáctica, 28 (1), 115-138.

Pérez, M. T.; Martín, M. A.; Arratia, O. y Galisteo, D. (2008) Innovación en docencia universitaria con Moodle. Casos prácticos. Alicante, Editorial Club Universitario.

Pou, J. (2010) El pediatra y el maltrato infantil. Madrid, Editorial Ergon.

Romero, C. y Pereira, C. (2011) El enfoque positivo de la educación: aportaciones al desarrollo humano. Teoría de la Educación. Revista Interuniversitaria, 23 (2), 69-89.

SHEPARD, S. y BREW, L. (2005) Teaching Theories of Couples Counseling: The Use of Popular Movies. The Family Journal, 13 (4), 406-415.

SHORTLAND, M. (1987) Screen Memories: Toward a History of Psychiatry and Psychoanalysis in the Movies. British Journal for the History of Sciencie, 20 (4), 421-452.

Solé, J.; GÓmez, M. y Gimeno, X. (2007) Tres propuestas formativas a través del cine. Sleepers como estudio de caso. Universitas Tarraconensis, III época, 33-59.

STAKe, R. E. (1995) The Art of. Thousand Oaks, Sage.

- (1998) Investigación con estudios de caso. Madrid, Morata.

Touriñán, J. M. (dir.) (2010) Artes y educación. Fundamentos de Pedagogía mesoaxiológica. La Coruña, Netbiblo.

UbiETo, J. R. (2012) La construcción del caso en el Trabajo en Red. Barcelona, Editorial uOc.

VARGAS, J. e IBÁÑ̃Z, E. (2008) Grupos focales en la evaluación de materiales didácticos para la educación superior. Revista Electrónica de Psicología Iztacala, 11 (3). Consultado el 31 de marzo del 2011. http://www.iztacala.unam.mx/carreras/psicologia/psiclin/ vol11num3/Vol11No3Art4.pdf.

WinNicotT, D. (1965) El proceso de maduración en el niño. Barcelona, Laia.

- (1982) Realidad y juego. Barcelona, Gedisa.

YIN, R. K. (1989) Case study research: Design and methods. Newbury Park Ca: Sage Publications. 